\title{
Rydberg excitation of Bose-Einstein condensates
}

\author{
Rolf Heidemann $* *$ Ulrich Raitzsch, Vera Bendkowsky, Björn Butscher, Robert Löw, and Tilman Pfau \\ 5. Physikalisches Institut, Universität Stuttgart, Pfaffenwaldring 57, 70569 Stuttgart, Germany
}

(Dated: 29 October 2007)

\begin{abstract}
Rydberg atoms provide a wide range of possibilities to tailor interactions in a quantum gas. Here we report on Rydberg excitation of Bose-Einstein condensed ${ }^{87} \mathrm{Rb}$ atoms. The Rydberg fraction was investigated for various excitation times and temperatures above and below the condensation temperature. The excitation is locally blocked by the van der Waals interaction between Rydberg atoms to a density-dependent limit. Therefore the abrupt change of the thermal atomic density distribution to the characteristic bimodal distribution upon condensation could be observed in the Rydberg fraction. The observed features are reproduced by a simulation based on local collective Rydberg excitations.
\end{abstract}

The ability to tailor interactions among atoms in a quantum gas is one of the main strengths of ultracold atoms as model systems for other research fields such as condensed matter physics. So far mostly effective contact interactions have been used which can be changed over a wide range in strength and even in sign. Two years ago, the long-range magnetic dipole-dipole interaction was observed between atoms in a ${ }^{52} \mathrm{Cr}$-BEC [1]. It was proposed to induce dynamic dipole-dipole interactions [2] or a $1 / r$ interaction potential [3] between ultracold atoms by light. To induce a static electric dipole-dipole interaction, it was suggested to admix a Rydberg state with a static electric dipole moment to the atomic ground state by photo-excitation [4].

Rydberg atoms and ground state atoms are predicted to form unusual weakly bound molecular states [5, $[$ ] $]$. As the average interparticle distances in BECs are of the same order of magnitude as the molecular binding distances, a BEC seems to be the ideal starting point for photoassociation of these molecules.

A Rydberg atom also constitutes an impurity in the BEC related to Ref. 7], which could be manipulated by electric fields. The interaction could lead to an agglomeration of ground state atoms around the impurity as was calculated for ionic impurities [8] .

As the nature and strength of the interaction among Rydberg atoms can be tailored by electric fields and microwave fields e.g. from van der Waals type to dipole dipole type [9] or an isotropic $1 / r^{3}$ potential [10] they provide a new tool for many body quantum physics.

In this Letter we describe the excitation of atoms to a Rydberg state while undergoing a phase transition from a thermal gas to a Bose-Einstein condensate. We present a model based on collective excited states to simulate the observed Rydberg fraction across the phase transition which agrees qualitatively with the observed data.

Using the setup described in [11], we magnetically trap ${ }^{87} \mathrm{Rb}$-atoms in the $5 \mathrm{~S}_{1 / 2}, F=2, m_{F}=2$ state and produce samples from thermal clouds to Bose-Einstein condensates by means of forced RF-evaporation. We vary the temperature from $5 \mu \mathrm{K}$ down to $200 \mathrm{nK}$ crossing the condensation temperature at around $700 \mathrm{nK}$. After this preparation, the atoms are subject to a two-photon Rydberg excitation via the $5 \mathrm{P}_{3 / 2}$ state to the $43 \mathrm{~S}_{1 / 2}$ state. The duration of the square pulses of excitation light was varied in this experiment between $170 \mathrm{~ns}$ and $2 \mu \mathrm{s}$. To avoid significant absorption and heating due to spontaneous photon scattering, the light is blue detuned by $\Delta=$ $483 \mathrm{MHz}$ from the $5 P_{3 / 2}, F=3$ level. Thus only one photon per 100 atoms is scattered for the longest excitation time. For the following experiments the Rabi frequency $\Omega_{1}$ on the $5 \mathrm{~S}-5 \mathrm{P}$ transition is $11 \mathrm{MHz}$. A Rabi frequency $\Omega_{2}$ for the $5 \mathrm{P}-43 \mathrm{~S}$ transition of $9.7 \mathrm{MHz}$ [12] results in a two-photon Rabi frequency $\Omega_{1} \Omega_{2} /(2 \Delta)$ of $110 \mathrm{kHz}$. The thermal motion of the atoms is negligible on the $\mu$ s-time scale of the experiments, but interactions among the Rydberg atoms can lead to collisions and ionisation. By choosing a Rydberg state with repulsive interactions and applying an electric extraction field, the unwanted effects of ions are avoided [13].

The Rydberg excitation is followed immediately by a field-ionisation of the Rydberg atoms and their detection on a microchannel plate. Up to three such sequences of excitation and detection are applied to one sample followed by absorption imaging of the remaining ground state atoms. From these calibrated detection methods, the total atom number $N_{g}$ and the fraction $f=N_{R} / N_{g}$ of atoms in the Rydberg state are derived. This normalisation of the Rydberg atom number $N_{R}$ is useful since the total atom number drops significantly from $10^{7}$ to $10^{5}$ while reducing the temperature. The temperature of each sample is derived from the size on the absorption image after an expansion during $20 \mathrm{~ms}$ time of flight. The thermal clouds are fitted using a Bose-distribution neglecting interaction among the ground state atoms. The BEC component is fitted with a Thomas-Fermi distribution. From the parameters of the fit and the known trapping potential, the in-trap density distributions are calculated and used for simulating the Rydberg excitation. The peak density and the condensate fraction are plotted versus temperature in Fig. 1 where no effect of the different excitation times on the atomic distribution is visible. The bimodality of the density distribution will prove to be crucial for the understanding of the results 

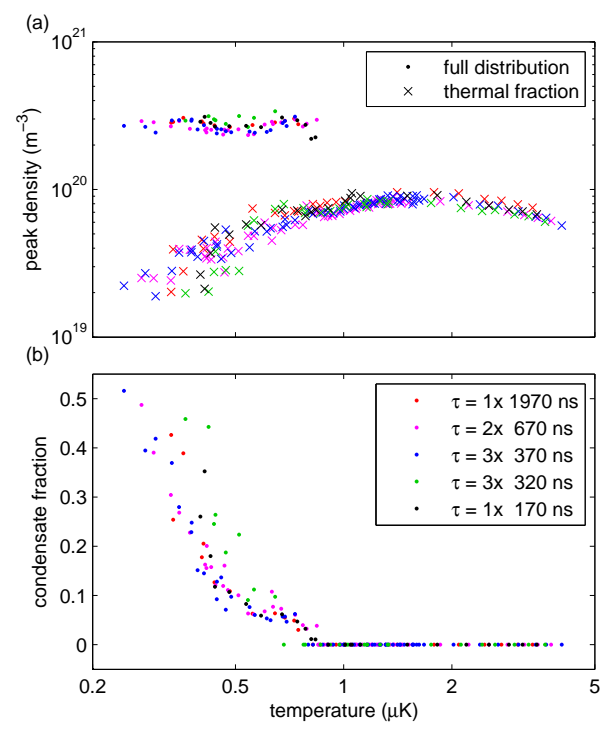

FIG. 1: (a) Dependence of the peak density of ground state atoms on the temperature. The crosses $(x)$ indicate the peak density of the thermal component, the dots $(\cdot)$ the peak density of the bimodal distribution (where a BEC component exists). In (b) the condensate fraction is plotted versus the temperature. The colors indicate the length of the Rydberg excitation pulse, which was applied in trap before taking the absorption picture after a time of flight of $20 \mathrm{~ms}$. The number of identical excitations within one sample is denoted by the multiplier in the legend. Note that no systematic effect of different excitation times on the distributions is visible. The same samples delivered the data presented in Fig. 2(a).

of the Rydberg excitation. When the temperature is decreased below the critical temperature, the central density increases abruptly by a factor of about 4 . When the temperature is reduced further, more atoms are condensed into the small constricted region of the BEC reducing the density of the thermal component. In the condensate the average atomic distance to the next neighbour is $150 \mathrm{~nm}\left(\approx 0.55 n_{g}^{-1 / 3}[14]\right)$ which is calculated from the peak atomic density $n_{g}$.

In a recent work, we investigated the density dependence of the Rydberg excitation dynamics in magnetically trapped clouds above the condensation temperature [13]. The Rydberg atom number as a function of excitation time initially increases linearly with a slope $R$ and saturates to a value $N_{\text {sat }}$ after a time $\tau_{s}=N_{\text {sat }} / R$. From this previous experimental observation we know that the saturation is reached faster with increasing density and that the saturation Rydberg density depends only very weakly on the density of ground state atoms $n_{g}$. In this Letter we investigate the excitation dynamics across the condensation temperature. Due to the bimodal density distribution in a partially condensed cloud the time scales and saturation Rydberg fractions are different in the condensate and thermal component. Figure

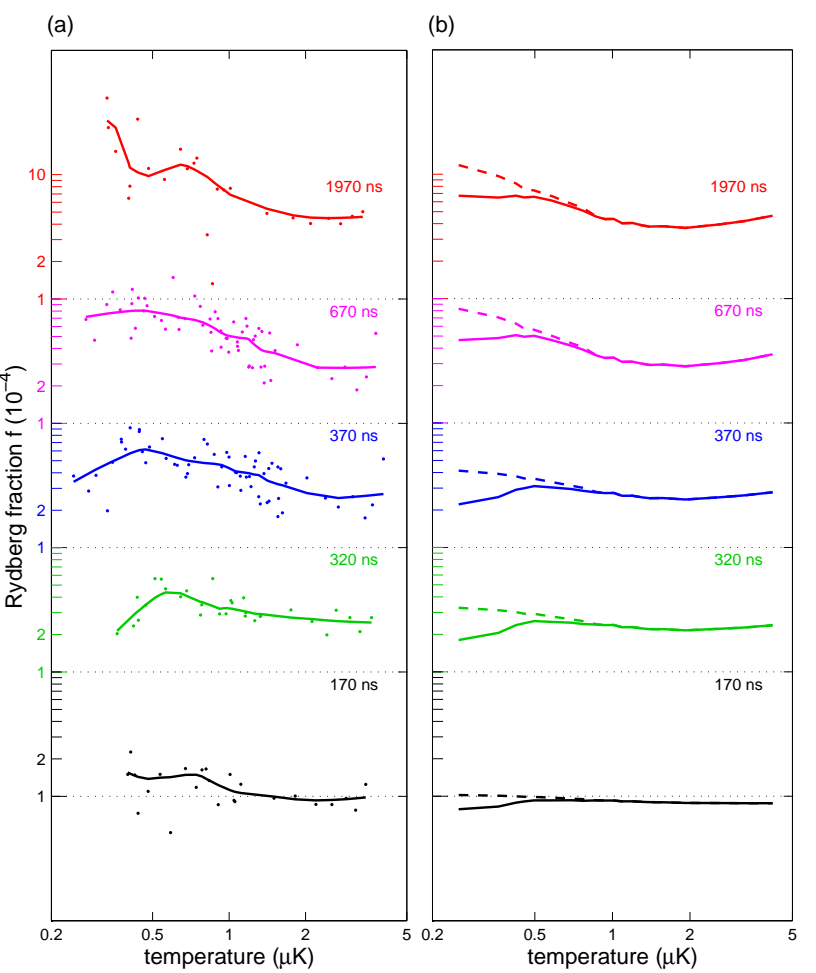

FIG. 2: Rydberg fraction as a function of temperature for different excitation times. The data sets for each excitation time are color-coded and for clarity shifted such that the dotted horizontal lines each indicate $10^{-4}$. The measurements in (a) are smoothed for each excitation time giving the solid lines as a guide to the eye [15]. The solid lines in (b) show the calculated Rydberg fractions based on the superatom model. The dashed lines show the result of the simulation taking only the thermal part of the atomic cloud. This confirms the BoseEinstein condensation as the origin of the kink in the Rydberg fraction.

2(a) shows the Rydberg fraction after different excitation times as a function of temperature. The lines are the result of a smoothing intended to guide the eye to the main features of the measurement: At medium excitation times (320 and $370 \mathrm{~ns}$ ) the Rydberg fraction decreases with proceeding formation of the condensate i.e. reduced temperature. For the shortest and longest excitation times, no such kink in the Rydberg fraction is visible at the critical temperature.

The excitation times in this experiment are chosen such that for the shortest time (170 ns), both components are just slightly blocked but not saturated. For longer excitation times, the excitation is already saturated in the condensate component while it is not yet in the thermal part. For the longest excitation time (1970 ns), the excitation is saturated in both components. As a start the following intuitive picture may be used to explain the reduction of the total Rydberg fraction at medium excitation times: With increasing BEC fraction, more atoms are condensed into the BEC where the Rydberg fraction 
is significantly lower than in the thermal cloud due to its higher density, this also lowers the total Rydberg fraction. This effect dominates over the smaller increase in the Rydberg fraction in the thermal cloud as its density decreases (see Fig.1(a)). A deeper comprehension is obtained by the following model based on collective states.

In this experiment the van der Waals interaction among the Rydberg atoms blocks further excitation within a blockade radius $r_{b}$ around one Rydberg atom [13, 16, 17]. This limitation to one excitation per blockade sphere leads to a collective Rabi oscillation which is enhanced in frequency by a factor $\sqrt{N}$. In this sense the $N$ atoms per blockade sphere act like a 'superatom' [18] with a $\sqrt{N}$ larger transition matrix-element. In the regime where the size of the sample is larger than the blockade radius $r_{b}$, quantum correlations interconnect the whole sample. Thus the system with $N_{g} \approx 10^{7}$ atoms and $N_{R} \approx 10^{3}$ excitations is inaccessible to direct ab initio simulations. The model described below reduces the quantum correlations to spatial correlations and locally collective states by assuming $N_{R}$ independent superatoms which oscillate at their respective collective Rabi frequencies $\sqrt{N} \Omega_{0}$. We will assume the superatoms to arrange in the close packing of a face-centered cubic structure with 12 next neighbours. In this hypothetical lattice, the saturation density is $n_{R}=\sqrt{2} r_{b}^{-3}$ and the interaction energy at the center of one superatom is $Z=14.5$ times the pairwise van der Waals ernergy. $Z$ is slightly larger than 12 due to the atoms beyond the shell of next neighbours. In a local density approximation the local number of ground state atoms forming one superatom is given by $N(r)=n_{g}(r) / n_{R}(r)$ and density variations on the scale of $r_{b}$ are neglected. The saturation density of Rydberg atoms can be derived from the blockade condition which equates the van der Waals interaction energy with the collective Rabi frequency, where $\kappa$ is a constant expected to be on the order of one:

$$
\frac{Z C_{6}}{r_{b}^{6}(r)}=Z C_{6} \frac{1}{2} n_{R}^{2}(r)=\kappa \hbar \sqrt{N(r)} \Omega_{0} .
$$

The $C_{6}$-coefficient for the $43 S$-state is $-1.7 \times 10^{19}$ a.u. [19]. As shown later, the blockade radius is in the micrometer regime, substantially larger than the mean interatomic distance. At these interatomic separations, the van der Waals interaction that is used in Eq. (11) is justified. From this relation, the local saturation density $n_{R}(r)$ and then the collective Rabi frequency $\Omega_{c}$ can be directly derived.

$$
\begin{aligned}
& n_{R}(r)=\left(2 \kappa \hbar / Z C_{6}\right)^{2 / 5} n_{g}^{1 / 5}(r) \Omega_{0}^{2 / 5}, \\
& \Omega_{c}(r)=\sqrt{N(r)} \Omega_{0}=\left(Z C_{6} / 2 \kappa \hbar\right)^{1 / 5} n_{g}^{2 / 5}(r) \Omega_{0}^{4 / 5}
\end{aligned}
$$

To illustrate the calculated bimodality in the saturation density of Rydberg atoms $n_{R}(r)$, Fig. 3 shows the density distributions as well as the distribution of the
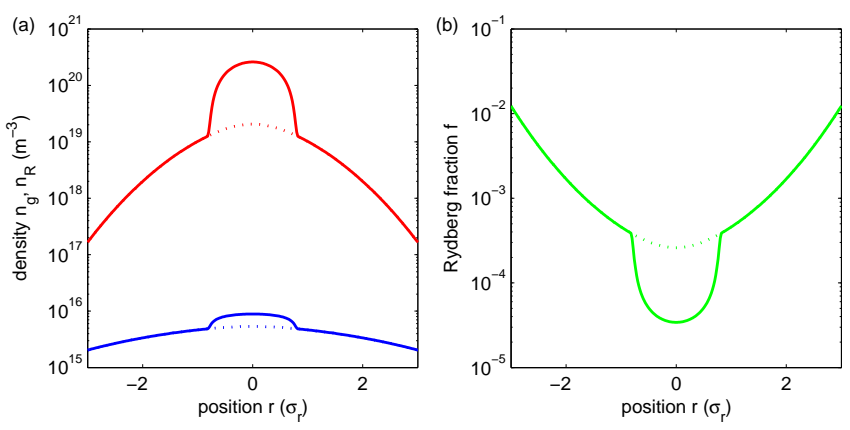

FIG. 3: In (a) the radial cross sections of the density distributions are shown for the ground state atoms $n_{g}(r)$ in red/light grey and for the simulated saturation density of Rydberg atoms $n_{R}(r)$ in blue/dark grey. The cross section of the local Rydberg fraction $f(r)$ is shown in (b). Each figure shows the calculated distributions for the lowest temperature of Fig. 2 and the dotted lines represent the thermal component without BEC. As can be seen, $f(r)$ decreases with $n_{g}(r)$. Therefore in the BEC $f$ is substantially lower than in the thermal cloud.

fraction $f(r)$ which shows a significant reduction at the position of the BEC component. The time dependence of the total number $N_{R}(\tau)$ of Rydberg atoms can be obtained by integrating the oscillations of the superatoms over the sample:

$$
N_{R}(\tau)=\int n_{R}(r) \cdot \sin ^{2}\left(\Omega_{c}(r) \tau / 2\right) d^{3} r
$$

The simulated behaviour of $N_{R}(\tau)$ nicely reproduces the saturation curves shown in Ref. [13]. The derived scaling of $N_{\text {sat }} \propto n_{R} \propto n_{g}^{1 / 5} \Omega_{0}^{2 / 5}$ with Rabi frequency is in excellent agreement with our previous observation where we found $N_{\text {sat }} \propto n_{g}^{0.07 \pm 0.02} \Omega_{0}^{0.38 \pm 0.04}[13]$, the scaling with the peak density $n_{g}$ is close. The calculated scalings of the initial slope of the excitation curve, $R \propto n_{R} \Omega_{c} \propto$ $n_{g}^{3 / 5} \Omega_{0}^{6 / 5}$, are in good agreement with the previous observation of $R \propto n_{g}^{0.49 \pm 0.06} \Omega_{0}^{1.1 \pm 0.1}$ [13]. This simplified model, despite its approximate character, explained well the observed scalings. Here we apply it for the situation of a changing density distribution $n_{g}(r)$ upon condensation. The lines in Fig.2(b) show the calculated values for the Rydberg fraction using this model. The atomic density distributions were calculated from the information obtained from the absorption images and averaged over several pictures taken at different excitation times. The simulation uses two free parameters which is the propotionality factor $\kappa$ in Eq. (1) and the Rabi-frequency $\Omega_{0}$. The factor $\kappa$ was adjusted to 0.3 such that the measurement is best reproduced for high temperatures. The Rabi frequency had to be reduced by a factor of 5.5 to reproduce the characteristic kink at the right excitation times. This reduction is reasonable as the frequency of the excitation lasers is only stable within $1.5 \mathrm{MHz}$ between adjacent excitations thus effectively reducing the Rabi-frequency in this model. Note that the simulation 
was used beyond the large radius $r$, where the model gets unphysical due to $n_{R}(r)>n_{g}(r)$. For our parameters the effect on the total fraction $f$ is negligible as only a fraction of at most $10^{-4}$ of the atoms are at these large distances where the collective behaviour ends and their single-atom excitation fraction is negligible.

The scaling of the Rydberg fraction $f=N_{R} / N_{g}$ with density can be derived from the model for short, moderate and long excitation times: For short excitation times, when the dynamics cannot yet be distinguished from single-atom behaviour, the Rydberg atom number is proportional to $N_{g}$, therefore $f$ is independent of $n_{g}$. For intermediate excitation times $\tau$, during the linear increase of $N_{R}(\tau), f$ can be approximated by $R \tau / N_{g} \propto$ $n_{R} \Omega_{c} \tau / n_{g} \propto n_{g}^{-2 / 5}$. For long excitation times, in the saturation regime, the dependence of the fraction on the density is stronger: $f \propto N_{\text {sat }} / N_{g} \propto n_{R} / n_{g} \propto n_{g, 0}^{-4 / 5}$. These considerations hold for a change in density without changing the shape of the distribution i.e. without crossing the critical temperature or for the thermal component alone.

The simulation is able to reproduce the main features of the experimental data: The dependence of $f$ on the density is increasing for increasing excitation time. For intermediate excitation times, $f$ bends down below the condensation temperature. At long excitation times, this kink is covered by the increased dependence of $f$ on $n_{g}$ in the saturated thermal cloud. To emphasize the effect of the bimodal density distribution, the dashed lines in Fig.2(b) show the calculation which includes only the thermal component of the atomic cloud which does not reproduce the characteristic kink. According to this simulation, the maximum density of Rydberg atoms in the BEC is $9 \times 10^{15} \mathrm{~m}^{-3}$, corresponding to a blockade radius of $5.4 \mu \mathrm{m}$ and 5 Rydberg excitations within the volume of the BEC.

The experimental fraction $f$ shows an overall increase with decreasing temperature which is not sufficiently reproduced by the simulation. We attribute this remaining discrepancy to a failure of the local density approximation (LDA). The radial width of the gaussian distribution gets as narrow as $2 \mu \mathrm{m}$ while the dynamics depends on a density that is averaged over the size of the blockade radius. For low temperatures this averaging effectively lowers the ground state densities and leads to higher Rydberg fractions than the simulation suggests.

Note that we do not expect the change in the densitydensity correlation upon condensation to have a significant effect on the described measurements as the de Broglie wavelength which is the length scale on which the bunching in the thermal cloud occurs is on the order of $(\lesssim 350 \mathrm{~nm})$ and therefore much smaller than the blockade radius.

To conclude, we have demonstrated the Rydberg excitation of Bose-Einstein condensates and show that the momentum distribution of the condensate is not significantly affected by the presence of the Rydberg atoms. We present measurements on the excitation dynamics which show a significantly smaller Rydberg fraction in the condensed sample than in the thermal sample. A simplified superatom model reproduces the main features of the measurement as a consequence of the changing density distribution upon condensation. The coherence of the condensate was not relevant for the understanding of the measurement of the overall excitation dynamics. Future studies will focus on making use of the coherence properties of the condensate, e.g. in order to measure the spatial density-density correlation function of the Rydberg atoms by means of matter wave interferometry.

We acknowledge fruitful discussions with F. Robicheaux, H.P. Büchler and L. Santos as well as financial support from the Deutsche Forschungsgemeinschaft within the SFB/TRR21 and under the contract PF 381/4-1, U.R. acknowledges support from the Landesgraduiertenförderung Baden-Württemberg.

* Electronic address: r.heidemann@physik.uni-stuttgart.de

$\dagger$ Electronic address t.pfau@physik.uni-stuttgart.de

[1] J. Stuhler, et al., Phys. Rev. Lett. 95, 150406 (2005).

[2] R. Löw, R. Gati, J. Stuhler, and T. Pfau, Europhys. Lett 71, 214 (2005).

[3] D. O'Dell, S. Giovanazzi, G. Kurizki, and V. M. Akulin, Phys. Rev. Lett. 84(25), 5687 (2000).

[4] L. Santos, G. V. Shlyapnikov, P. Zoller, and M. Lewenstein, Phys. Rev. Lett. 85, 1791 (2000).

[5] C. H. Greene, A. S. Dickinson, and H. R. Sadeghpour, Phys. Rev. Lett. 85(12), 2458 (2000).

[6] C. H. Greene, et al., Phys. Rev. Lett. 97, 233002 (2006).

[7] A. P. Chikkatur, et al., Phys. Rev. Lett. 85, 483 (2000).

[8] P. Massignan, C. J. Pethick, and H. Smith, Phys. Rev. A 71(2), 023606 (2005).

[9] T. Vogt, et al., Phys. Rev. Lett. 99(7), 073002 (2007).

[10] H. P. Büchler, A. Micheli, and P. Zoller, Nature Physics 3, 726 (2007).

[11] R. Loew, et al., eprint arXiv.org:0706.2639 (2007).

[12] This value is estimated from our calculation of the dipole matrix element, which is in excellent agreement with an independent calculation of F. Robicheaux (priv. comm.).

[13] R. Heidemann, et al., Phys. Rev. Lett. 99, 163601 (2007).

[14] P. J. Clark and F. C. Evans, Ecology 35, 445 (1954).

[15] The smoothing was done with a robust locally weighted linear regression method (lowess), using the $50 \%$ nearest data points at a time for regression [20].

[16] D. Tong, et al., Phys. Rev. Lett. 93(6), 063001 (2004).

[17] K. Singer, et al., Phys. Rev. Lett. 93(16), 163001 (2004).

[18] V. Vuletic, Nature Physics 2, 801 (2006).

[19] K. Singer, J. Stanojevic, M. Weidemüller, and R. Côté, J. Phys. B: At., Mol. Opt. Phys. 38, S295 (2005).

[20] W. Cleveland, J. Amer. Statist. Assoc. 74(368), 829 (1979). 